\title{
Acute or Subacute Infectious Encephalitis: How to Approach?
}

\section{Rajib Dutta*}

MD Neurology, India

*Corresponding Author: Rajib Dutta, MD Neurology, India.

E-mail: rajibdutta808@gmail.com

ORCID ID: http://orcid.org/0000-0002-6129-1038

DOI: 10.31080/ASMI.2020.03.0527
Received: February 08, 2020

Published: February 14, 2020

(C) All rights are reserved by Rajib Dutta.

\section{Abstract}

Encephalitis is a condition characterized by inflammation of brain parenchyma leading to symptoms like acute fever, seizures, altered mental status, focal neurological deficits, neurological disability post recovery and even death. Infectious cause may comprise of virus, bacteria, fungus or parasitic agents. Viral causes account for the largest proportion of cases but encephalitis caused by microbes other than virus are frequently reported in immunocompetent individuals. This review article focuses on diagnosis, management and prognosis of acute or subacute infectious causes of encephalitis. Role of cerebrospinal fluid studies, MRI, novel diagnostic methods like next generation sequencing are also studied.

Keywords: Encephalitis; Infection; Immunocompetent; Diagnosis; Management; Prognosis; Next Generation Sequencing

\section{Abbreviations}

TLRS: Toll-Like Receptors; RIG-I: Retinoic Acid-Inducible Gene 1; HLA: Human Leukocyte Antigen; PCR: Polymerase Chain Reaction; RT-PCR: Reverse Transcription Polymerase Chain Reaction; CNS: Central Nervous System; MTB: Mycobacterium Tuberculosis; RIF: Resistance to Rifampicin; HIV: Human Immunodeficiency virus; IgM: Immunoglobulin M; IgG: Immunoglobulin G

\section{Introduction}

Encephalitis is a serious condition characterized by altered mental status along with various combinations of acute fever, seizures not attributable to a pre-existing seizure disorder, focal neurologic deficits of recent onset, psychosis, cerebrospinal fluid (CSF) pleocytosis, neuroimaging and electroencephalographic (EEG) abnormalities [1]. It is the inflammation of the brain parenchyma caused by wide variety of infectious and autoimmune causes where new onset neuropsychiatric symptoms and short term memory loss can occur in later [2]. Alternative pathological processes in brain, metabolic and systemic causes should be ruled out while suspecting diagnosis of encephalitis because of overlap of conditions $[3,4]$. Time of onset and progression should be used meticulously to differentiate acute encephalitis from other neurodegenerative conditions which can lead to rapid progressive dementia [5]. However, diagnostic certainty during presentation increases with increasing numbers of supportive features [1].

Up to $12 \cdot 6$ per 100000 individuals are affected by encephalitis annually, with the highest incidence in children [6-9]. The cause is unknown in approximately $50 \%$ of these cases which causes unnecessary confusion among clinicians while treating patients. Of the cases with a known cause, 20 to $50 \%$ are attributed to viruses $[8,10]$, followed by acute disseminated encephalomyelitis (ADEM), a post-infectious or para-infectious condition. Of viral causes, Japanese encephalitis virus (JEV) is the most commonly identified epidemic cause and herpes simplex virus (HSV) the most commonly identified sporadic cause followed by varicella-zoster virus (VZV), enteroviruses, and arboviruses. In recent times because of the emergence and spread of arthropod-borne viruses such as Zika and chikungunya, and the increasing use of vaccines (e.g. against JEV and varicella zoster virus) in developed and developing countries epidemiology has changed somewhat making it difficult at times [11]. 
HSV encephalitis occurs in all age groups and does not have any characteristic seasonal or geographic pattern, however encephalitis caused by arbovirus has considerable year-to-year variation in case counts, seasonal occurrence, and variation of incidence according to geographic region, suggesting about the ecology of arboviral transmission. Bacterial, fungal and parasitic infection can also cause encephalitis however presentation can be broad depending on the neurotropicity of the microorganisms to specific brain parts like brainstem or cerebellar etc.

Factors that affect susceptibility of pathogens causing encephalitis are poorly understood. Certain organisms cause central nervous system disease predominantly in children, whereas others tend to cause severe central nervous system disease in the elderly. However, HSV can cause encephalitis in persons at both ends of the age spectrum. Host factors which is responsible for protection and fighting against the pathogen depends on innate and adaptive immunity of the individual. Age-related changes includes reduced expression of toll-like receptors (TLRs) and retinoic acid-inducible gene 1 (RIG-I)-like receptors, decreased phagocytic function, and reduced natural killer and cytotoxic T-cell activity, which may contribute to susceptibility in elderly persons $[12,13]$. On the contrary, children may have decreased type I interferon signaling, as compared with adults which may make them highly susceptible to organisms [14].

Variation in HLA regions, potentially affecting the efficiency of adaptive immune responses, have been associated with susceptibility to infection with herpesviruses and arboviruses $[13,15,16]$. Genetic factors in host involved in susceptibility to neurotropic viruses may be due to polymorphic sets of genes that influence both innate and adaptive immunity [17-19]. A loss-offunction deletion in chemokine receptor 5 results in impairment of lymphocyte trafficking into the central nervous system, which results in enhanced susceptibility to both tickborne encephalitis virus [20] and West Nile virus [21]. Mutations or polymorphisms in genes encoding components of innate immune pathways -notably, TLR3 and interferon signaling - have been linked to encephalitis caused by HSV in children, VZV-associated encephalitis, the measles-subacute sclerosing panencephalitis complex, Japanese encephalitis virus, enterovirus 71 and influenza A virus-associated encephalopathy [22-24]. Genome wide association studies have also linked polymorphisms in interferon signaling with an increased risk of initial infection and symptomatically severe West Nile virus encephalitis [25].

Encephalitis can be divided into Limbic encephalitis, Brainstem encephalitis, Multifocal encephalitis, and other syndromes. Limbic encephalitis can be typical of HSV, varicella zoster virus (VZV), syphilis, and rarely Mycobacterium tuberculosis [26]. Brainstem encephalitis include Listeria, Brucella, arthropod-borne viruses, enterovirus 71, associated with autonomic dysfunction, which results in fluctuating blood pressure and neurogenic pulmonary oedema with haemorrhage [27]. Multifocal encephalitis usually by arthropod-borne viruses and enteroviruses can present with multifocal involvement of the CNS, especially the deep grey matter. Tremors and other movement disorders like myoclonus or athetosis in patients who rapidly lose consciousness is characteristic for encephalitis caused by arthropod-borne viruses, including flaviviruses (e.g. JEV) and alphaviruses (e.g. eastern equine encephalitis virus). These virus along with enterovirus can attack the anterior horn cells of the spinal cord and cause acute flaccid paralysis, which could be part of an encephalitis syndrome $[27,28]$, mimicking poliomyelitis like presentation.

Organisms involved in acute to subacute infectious encephalitis

Viral

Global sporadic

- Herpesviridae: Herpes simplex virus type 1, Herpes simplex virus type 2, Varicella zoster virus, Epstein-Barr virus, Human herpes virus type 6, Human herpes virus type 7.

- Picornaviridae: Enterovirus type 70 and 71, Coxsackie virus, Poliovirus

- Paramyxoviridae: Measles virus, Mumps virus.

- Orthomyxoviridae: Influenza virus.

\section{Geographically-restricted}

- Flaviviridae: Japanese encephalitis virus, Dengue virus, West Nile virus, Zika virus, St Louis encephalitis virus, Murray Valley encephalitis virus, Tick-borne encephalitis virus, Powassan virus.

- Bunyaviridae: La Crosse virus, Toscana virus, Jamestown Canyon virus, California encephalitis virus 
- Togaviridae: Chikungunya virus, Eastern and Western equine encephalitis viruses, Venezuelan equine encephalitis virus

- Paramyxoviridae: Nipah virus.

- Rhabdoviridae: Rabies virus.

- Coltivirus: Colorado tick fever virus [30,67-69].

\section{Bacterial}

Bartonella spp, Borrelia spp, Brucella spp, Listeria monocytogenes, Mycobacterium tuberculosis, Mycoplasma pneumoniae, Rickettsia, Ehrlichia, and Anaplasma, Treponema pallidum [68,69].

Fungal

Blastomyces dermatides, Coccidiodes immitis, Cryptococcus spp, Histoplasma capsulatum [68,69].

Parasitic

Acanthamoeba spp, Balamuthia mandrillaris, Baylisascaris procyonis, Naegleria fowleri [68,69].

\section{Clinical presentation}

Clinical presentation can be complex with overlap of signs and symptoms. Careful medical history is mandatory especially the class of encephalitis (infectious or autoimmune), specific causes and syndromes and extra bits and pieces like parkinsonism, myoclonus, chorea, epilepsy etc. associated with it. The history should include consideration of the season during which the patient became ill, travelling, exposures (e.g. to animals, health of relatives, contact with sick persons, and known cases of encephalitis in the area), occupation, hobbies, recreational activities, diet, sexual practices, drug use, and health status (vaccinations, medical conditions and medications, and possible immunosuppression due to human immunodeficiency virus [HIV], chemotherapy, radiotherapy or cancer) to contextualise the acute encephalitis presentation and guide the differential diagnosis. Common causes of encephalitis are associated with distinguishing clinical features. The differential approach is based on the anatomy of the brain which in turn can give us the cause of the encephalitis. The physical and neurologic examinations may provide clues to potential causes and may guide testing. The presence of exanthems or skin lesions can give us indirect explanation or causative organisms may be helpful in identifying some forms of viral encephalitis but does not have high specificity $[1,4,29,30]$.
Diagnosis and investigation

To establish the diagnosis and find out the specific cause, assessment of the CSF, MRI brain, ancillary investigations of blood and other samples like urine and electroencephalography (EEG) is essential. Further testing in the form of PCR or RT-PCR of various samples may be tailored to reflect demographics, exposure, immune status, initial CSF, and imaging findings. HSV encephalitis is usually characterized by more pronounced CSF pleocytosis and more frequent focal abnormalities on EEG and neuroimaging as compared to non-HSV encephalitis [31]. Most neurologic symptoms, including impaired consciousness, confusion, aphasia, hallucinations, and movement disorders, do not differ among the various types of encephalitis [26].

Profiles of viral encephalitis can be divided into focal and generalized. Focal profile consists of temporal lobe, cerebellar, extrapyramidal or movement disorders due to thalamic or basal ganglia lesions and hydrocephalus. Generalized profile comprises of multifocal white-matter lesions, intractable seizures, newonset psychosis, diffuse cerebral edema, recurrent or chronic inflammatory CNS disease, seizures with rapid recovery [26,32,33]. CSF analysis reveals moderate elevation in the white cell count in most viral cases, commonly $10-200$ cells per $\mu \mathrm{L}^{3}$, although up to 800 cells per $\mu \mathrm{L}^{3}$ has been reported in HSV encephalitis [34]. In addition, glucose is typically normal and protein is modestly elevated. When the CSF white cell count is $>1000$ cells per $\mu \mathrm{L}^{3}$, protein is $>1 \mathrm{~g} / \mathrm{L}$, or CSF glucose level is less than two-thirds of serum levels, a non-viral infectious aetiology (i.e. bacterial, mycobacterial, or fungal) should be strongly considered. Although, a lymphocytic pleocytosis is characteristic of viral encephalitis, neutrophils might predominate early in viral infection, and 3 - 26\% with HSV encephalitis have no pleocytosis in early CSF samples $[34,35]$.

Repeat CSF $24-72 \mathrm{~h}$ after the first lumbar puncture should be considered if clinical suspicion of encephalitis remains [36]. Eosinophils can be present in the setting of helminthic infections such as Baylis ascaris pryocyonis but can also be seen at lower frequency in fungal and mycobacterial encephalitis [37]. Because of the hemorrhagic nature of HSV encephalitis, red blood cells and xanthochromia can also be identified as a late manifestation and can also be found with VZV [38]. Although flavivirus RNA can be 
detectable in serum by RT-PCR during the viraemic phase or the first few days of neurological symptoms, most patients are diagnosed by the detection of specific IgM antibodies, demonstration of a four-fold rise in titre by other assays, or by intrathecal synthesis [39]. Encephalitis caused by Zika virus and West Nile virus, PCR of urine is helpful because it can remain positive after blood is cleared of the virus [40].

In Listeria monocytogenes encephalitis, a mild lymphocytic predominant pleocytosis with a normal to minimally elevated protein, similar to viral encephalitis is found, however, the CSF to serum glucose ratio is typically lower in Listeria than in viral infections. Multiple cultures from blood and CSF might be required to confirm the diagnosis [41]. The diagnosis of CNS tuberculosis in the form of encephalitis is a big challenge because the sensitivity of CSF smear is less than $25 \%$ and that of culture is less than $50 \%$ [42]. Extended examination of at least $5 \mathrm{mLs}$ of CSF may be required for identification and diagnostic certainty [43]. In 2017,WHO issued recommendations for use of the Xpert MTB/RIF assay as the initial test for diagnosis of tuberculous meningitis [44]. Cerebrospinal fluid testing for the ( 1 - 3)- $\beta$-D-glucan glycoprotein, a component of the cell wall of many fungi, has shown some promise although some limitations exist [45].

Abnormalities on T2-weighted and fluid-attenuated inversion recovery (FLAIR) sequences in the temporal lobes and to a lesser extent orbitofrontal and cingulate cortices are highly suggestive of HSV encephalitis particularly when asymmetrical [30]. Temporal lobe involvement can occur with HSV-1 or, less commonly, HSV2 and also with VZV $[26,46]$. Neurosyphilis can rarely present acutely with lesions in the temporal lobe in neuroimaging [26]. Deep grey matter abnormalities on T2/FLAIR can be seen in arboviral encephalitis, influenza-associated encephalitis and encephalopathy, or CNS tuberculosis [33]. Presence of enhancement of the basilar meninges and hydrocephalus, for example, suggests tuberculosis, fungal infections, or Balamuthia mandrillaris and if brainstem is suspected Listeria should be high in the differential. Evidence of haemorrhage can suggest HSV$1, \mathrm{VZV}$, influenza associated acute necrotising encephalopathy, arbovirus infection [47].

EEG on the other hand can be used to differentiate between infectious and autoimmune encephalitis when CSF and MRI findings are nondiagnostic. It can also be used to rule out any psychiatric diagnosis because of the overlap of symptoms [4] and also in patients with altered consciousness, where it can identify patients in subtle motor or non-convulsive status epilepticus, which might present de novo or evolve from a convulsive to nonconvulsive picture in the setting of encephalitis.

Dengue and chikungunya are also common cause of fever in returning travellers and are sometimes associated with neurological disease and clinicians should be cautious [48]. Hyponatraemia is found in many forms of encephalitis [49]. HIV antibody and RNA tests are important during the HIV seroconversion illness phase presenting as meningo-encephalitis along with other opportunistic pathogens which may present with CNS infections [4]. Viral culture or PCR of skin lesions (for VZV or enteroviruses), throat and rectal swabs (for enteroviruses) and respiratory samples (for influenza) should be considered in whom CSF and MRI might show evidence of inflammation of the CNS however no pathogen is diagnosed by PCR, culture, or IgG and IgM analysis of the CSF [50].

Recent advances in investigations include Bio Fire Film Array panel, a multiplex PCR-based system for detection of pathogens in the CSF [51,71], next generation sequencing in immunocompromised and pathogen identification in otherwise undiagnosed cases of encephalitis due to leptospira, Cache Valley virus, astrovirus, variegated squirrel bornavirus, parvovirus 4,St.Louis encephalitis virus, Powassan virus, hepatitis E virus, Balamuthia mandrillaris [52-54,72-75]. Bead-array and proteomic approaches to assess the host inflammatory responses i.e. chemokines and cytokines are increasingly being explored to investigate the pathophysiological processes that could be amenable to targeted immunomodulatory therapies $[55-58,70]$.

\section{Management and prognosis}

Suspected patients should undergo initial assessment of airway, breathing, circulation, blood sugar. Primary focus should be on seizures and raised intracranial pressure management, followed by target based empirical or specific therapy directed at the pathogen. HSV/VZV should be treated with acyclovir along with antibiotics if concurrent bacterial infection suspected. The frequency, morbidity and mortality is associated with delayed treatment of HSV encephalitis, empirical aciclovir $(10 \mathrm{mg} / \mathrm{kg}$ intravenously every $8 \mathrm{~h}$ in individuals with normal renal function) should be initiated and continued until the diagnosis of HSV has been excluded and should be continued for at least 2 weeks [59]. In children (3 months to 12 
years of age) with HSV encephalitis, a higher dose of acyclovir (20 mg per kilogram every 8 hours for 21 days) has been recommended, since this results in better outcomes and fewer relapses than lower doses [60]. Immunomodulatory agents like corticosteroids either an adjunct to antiviral drugs or as monotherapy are of uncertain benefit $[61,62]$. Overall, mortality rates for encephalitis range between approximately 5 - 15\% [63].

\section{Prevention}

Absence of proper treatment to neurotropic virus has strongly led to emphasis on prevention [64]. Effect of a 5-year vaccination campaign in Nepal for the prevention of Japanese encephalitis virus showed that cases of disease were reduced by 78\% [65]. A universal program of varicella virus vaccination for 1-yearold children in Germany in 2004 resulted in an estimated 60\% decrease in varicella-associated neurologic complications [66].

\section{Conclusion}

Infectious encephalitis is a major cause of illness, death and poses a huge economic burden to the society. Rapid deterioration and brainstem involvement points towards poor prognosis. Factors contributing to delays in drug administration have a negative impact on recovery. New diagnostic strategies and technologies should be studied to differentiate among different group of pathogens causing encephalitis. Treatment remains largely empirical and, with the exception of acyclovir for HSV encephalitis. New prevention strategies, inhibiting viral replication, proper and targeted use of antimicrobials are of prime importance.

\section{Acknowledgment}

The author has no relevant affiliations or financial involvement with any organization or entity with a financial interest in or financial conflict with the subject matter or materials discussed in the manuscript. Special thanks to my supervisor Professor Dr. Huifang Shang who gave initial ideas and supported me through this research study. I would also like to thank Dr. Swatilekha Roy Sarkar for her valuable feedback on the manuscript and literature screening from PubMed.

\section{Bibliography}

1. Venkatesan A., et al. "Case definitions, diagnostic algorithms, and priorities in encephalitis:consensus statement of the International Encephalitis Consortium". Clinical Infectious Diseases 57 (2013): 1114-1128.

2. Graus F., et al. "A clinical approach to diagnosis of autoimmune encephalitis". The Lancet Neurology 15 (2016): 391-404.
3. Venkatesan A and Geocadin RG. "Diagnosis and management of acute encephalitis: a practical approach". Neurology Clinical Practice 4 (2014): 206-215.

4. Solomon T., et al. "Management of suspected viral encephalitis in adults -Association of British Neurologists and British Infection Association National Guidelines". Journal of Infection 64 (2012): 347-373.

5. Geschwind MD., et al. "Rapidly progressive dementia". Annals of Neurology 64 (2008): 97-108.

6. Granerod J., et al. "Challenge of the unknown. A systematic review of acute encephalitis in non-outbreak situations". Neurology 75 (2010): 924-932.

7. Granerod J., et al. "New estimates of incidence of encephalitis in England". Emerging Infectious Diseases Journal 19 (2013): 1455-1462.

8. George BP., et al. "Encephalitis hospitalization rates and inpatient mortality in the United States, 2000-2010". PLoS One 9 (2014): e104169.

9. Boucher A., et al. "Epidemiology of infectious encephalitis causes in 2016". Médecine et Maladies Infectieuses 47 (2017): 221-35.

10. Vora NM., et al. "Burden of encephalitis-associated hospitalizations in the United States, 1998-2010". Neurology 82 (2014): 443-451.

11. Granerod J., et al. "Causes of encephalitis and differences in their clinical presentations in England:a multicentre, population-based prospective study". The Lancet Infectious Diseases 10 (2010): 835-844.

12. Montgomery RR. "Age-related alterations in immune responses to West Nile virus infection". Clinical and Experimental Immunology 187 (2017): 26-34.

13. Moraru M., et al. "Host genetic factors in susceptibility to herpes simplex type 1 virus infection:contribution of polymorphic genes at the interface of innate and adaptive immunity". The Journal of Immunology 188 (2012): 4412-4420.

14. Taylor KG., et al. "Age-dependent myeloid dendritic cell responses mediate resistance to La Crosse virus-induced neurological disease". Journal of Virology 88 (2014): 1107011079.

15. Long D., et al. "Identification of genetic variants associated with susceptibility to West Nile virus neuroinvasive disease". Genes and Immunity 17 (2016): 298-304. 
16. Crosslin DR., et al. "Genetic variation in the HLA region is associated with susceptibility to herpes zoster". Genes and Immunity 16 (2015): 1-7.

17. Qian F., et al. "Immune markers associated with host susceptibility to infection with West Nile virus". Viral Immunology 27 (2014): 39-47.

18. Qian F., et al. "Systems immunology reveals markers of susceptibility to West Nile virus infection". Clinical and Vaccine Immunology 22 (2015): 6-16.

19. Ignatieva EV., et al. "A database of human genes and a gene network involved in response to tick-borne encephalitis virus infection". BMC Evolutionary Biology 17 (2017): 259.

20. Mickiene A., et al. "Polymorphisms in chemokine receptor 5 and Toll-like receptor 3 genes are risk factors for clinical tickborne encephalitis in the Lithuanian population". PLoS One 9.9 (2014): e106798.

21. Lim JK., et al. "CCR5 deficiency is a risk factor for early clinical manifestations of West Nile virus infection but not for viral transmission". The Journal of Infectious Diseases 201 (2010): 178-185.

22. Mørk N., et al. "Mutations in the TLR3 signaling pathway and beyond in adult patients with herpes simplex encephalitis". Genes and Immunity 16 (2015): 552-566.

23. Verma R and Bharti K. "Toll like receptor 3 and viral infections of nervous system". Journal of the Neurological Sciences 372 (2017): 40-48.

24. Sironi M., et al. "TLR3 mutations in adult patients with herpes simplex virus and varicella-zoster virus encephalitis". The Journal of Infectious Diseases 215 (2017): 1430-1434.

25. Lim JK., et al. "Genetic variation in OAS1 is a risk factor for initial infection with West Nile virus in man". PLoS Pathogens 5.2 (2009): e1000321.

26. Chow FC., et al. "Use of clinical and neuroimaging characteristics to distinguish temporal lobe herpes simplex encephalitis from its mimics". Clinical Infectious Diseases 60 (2015): 1377-1383.

27. Ooi MH., et al. "Clinical features, diagnosis, and management of enterovirus 71". The Lancet Neurology 9 (2010): 1097-1105.

28. Solomon T., et al. "Poliomyelitis-like illness due to Japanese encephalitis virus". Lancet 351 (1998): 1094-1097.
29. Steiner I., et al. "Viral meningoencephalitis: a review of diagnostic methods and guidelines for management". European Journal of Neurology 17.8 (2010): 999-1009.

30. Tunkel AR., et al. "The management of encephalitis: clinical practice guidelines by the Infectious Diseases Society of America”. Clinical Infectious Diseases 47 (2008): 303-327.

31. Whitley RJ., et al. "Herpes simplex encephalitis". Journal of the American Medicine Association 247 (1982): 317-20.

32. Glaser CA., et al. "Beyond viruses: clinical profiles and etiologies associated with encephalitis". Clinical Infectious Diseases 43 (2006): 1565-1577.

33. Beattie GC., et al. "Encephalitis with thalamic and basal ganglia abnormalities: etiologies, neuroimaging, and potential role of respiratory viruses". Clinical Infectious Diseases 56 (2013): 825-832.

34. Sili U., et al. "Herpes simplex virus encephalitis: clinical manifestations, diagnosis and outcome in 106 adult patients". Journal of Clinical Virology 60 (2014): 112-118.

35. Raschilas F., et al. "Outcome of and prognostic factors for herpes simplex encephalitis in adult patients: results of a multicenter study". Clinical Infectious Diseases 35 (2002): 254-260.

36. Bradshaw MJ and Venkatesan A. "Herpes simplex virus-1 encephalitis in adults: pathophysiology, diagnosis, and management". Neurotherapeutics 13 (2016): 493-508.

37. MurrayWJ and Kazacos KR. "Raccoon roundworm encephalitis". Clinical Infectious Diseases 39 (2004): 1484-1492.

38. Kaewpoowat Q., et al. "Herpes simplex and varicella zoster CNS infections: clinical presentations, treatments and outcomes". Infection 44 (2016): 337-345.

39. David S and Abraham AM. "Epidemiological and clinical aspects on West Nile virus, a globally emerging pathogen". Infectious Diseases 48 (2016): 571-586.

40. Soares CN., et al. "Fatal encephalitis associated with Zika virus infection in an adult". Journal of Clinical Virology 83 (2016): 63-65.

41. Armstrong RW and Fung PC. "Brainstem encephalitis (rhombencephalitis) due to Listeria monocytogenes: case report and review". Clinical Infectious Diseases 16 (1993): 689-702. 
42. Lipsky BA., et al. "Factors affecting the clinical value of microscopy for acid-fast bacilli". Reviews of Infectious Diseases 6 (1984): 214-222.

43. Mai NT and Thwaites GE. "Recent advances in the diagnosis and management of tuberculous meningitis". Current Opinion in Infectious Diseases 30 (2017): 123-128.

44. Bahr NC., et al. "Diagnostic accuracy of Xpert MTB/RIF Ultra for tuberculous meningitis in HIV-infected adults: a prospective cohort study". The Lancet Infectious Diseases 18 (2018): 68-75.

45. Lyons JL and Zhang SX. "Current laboratory approaches to diagnosis of CNS fungal infections". Future Microbiology 11 (2016): 175-177.

46. Whitley RJ., et al. "Diseases that mimic herpes simplex encephalitis. Diagnosis, presentation, and outcome. NIAD Collaborative Antiviral Study Group". The Journal of the American Medical Association 262 (1989): 234-239.

47. Glaser CA and Venkatesan A. In: Scheld WWR, Marra CM, eds. Infections of the Central Nervous System. Philadelphia: Wolters Kluwer Health, (2014): 84-111.

48. Wilson ME., et al. "Fever in returned travelers: results from the GeoSentinel Surveillance Network". Clinical Infectious Diseases 44 (2007): 1560-1568.

49. Czupryna P., et al. "Comparison of hyponatremia and SIADH frequency in patients with tick borne encephalitis and meningitis of other origin". Scandinavian Journal of Clinical and Laboratory Investigation 76 (2016): 159-164.

50. Goenka A., et al. "Neurological manifestations of influenza infection in children and adults: results of a National British Surveillance Study". Clinical Infectious Diseases 58 (2014): 775-784.

51. Leber AL., et al. "Multicenter evaluation of biofire filmarray meningitis/encephalitis panel for detection of bacteria, viruses, and yeast in cerebrospinal fluid specimens". Journal of Clinical Microbiology 54 (2016): 2251-2261.

52. Brown JR., et al. "Encephalitis diagnosis using metagenomics: application of next generation sequencing for undiagnosed cases". Journal of Infectious Diseases 76 (2018): 225-240.

53. Simner PJ., et al. "Understanding the promises and hurdles of metagenomic next-generation sequencing as a diagnostic tool for infectious diseases". Clinical Infectious Diseases 66 (2018): 778-788.
54. Wilson MR., et al. "Diagnosing Balamuthia mandrillaris encephalitis with metagenomic deep sequencing". Annals of Neurology 78 (2015): 722-730.

55. Michael BD., et al. "The interleukin-1 balance during encephalitis is associated with clinical severity,blood-brain barrier permeability, neuroimaging changes,and disease outcome". The Journal of Infectious Diseases 213 (2016): 16511660 .

56. Griffiths MJ., et al. "In enterovirus 71 encephalitis with cardiorespiratory compromise, elevated interleukin $1 \beta$, interleukin 1 receptor antagonist, and granulocyte colony-stimulating factor levels are markers of poor prognosis". The Journal of Infectious Diseases 206 (2012): 881-892.

57. Bastos MS., et al. "Divergent cerebrospinal fluid cytokine network induced by non-viral and different viral infections on the central nervous system". BMC Infectious Diseases 15 (2015): 345.

58. Kothur K., et al. "Utility of CSF cytokine/chemokines as markers of active intrathecal inflammation: comparison of demyelinating, anti-NMDAR and enteroviral encephalitis". PLoS One 11.8 (2016): e0161656.

59. Whitley RJ., et al. "Vidarabine versus acyclovir therapy in herpes simplex encephalitis". The New England Journal of Medicine 314 (1986): 144-149.

60. Kimberlin DW., et al. "Safety and efficacy of high-dose intravenous acyclovir in the management of neonatal herpes simplex virus infections". Pediatrics 108 (2001): 230-238.

61. Maraş Genç H., et al. "Clinical outcomes in children with herpes simplex encephalitis receiving steroid therapy". Journal of Clinical Virology 80 (2016): 87-92.

62. Ramos-Estebanez C., et al. "A systematic review on the role of adjunctive corticosteroids in herpes simplex virus encephalitis: is timing critical for safety and efficacy?". Antiviral Therapy 19 (2014): 133-139.

63. Venkatesan A. "Epidemiology and outcomes of acute encephalitis". Current Opinion in Neurology 28 (2015): 277282.

64. Leibovitch EC and Jacobson S. "Vaccinations for neuroinfectious disease: a global health priority”. Neurotherapeutics 13 (2016): 562-570. 
65. Upreti SR., et al. "Updated estimation of the impact of a Japanese encephalitis immunization program with live, attenuated SA 14-14-2 vaccine in Nepal". PLOS Neglected Tropical Diseases 11.9 (2017): e0005866.

66. Streng A., et al. "Decline of neurologic varicella complications in children during the first seven years after introduction of universal varicella vaccination in Germany, 2005-2011". The Pediatric Infectious Disease Journal 36 (2017): 79-86.

67. Salimi H., et al. "Encephalitic arboviruses: emergence, clinical presentation, and neuropathogenesis". Neurotherapeutics 13 (2016): 514-534.

68. Olsen SJ., et al. "Infectious causes of encephalitis and meningoencephalitis in Thailand, 2003-2005". Emerging Infectious Diseases Journal 21.2 (2015): 280-289.

69. Venkatesan A., et al. "Acute encephalitis in immunocompetent adults". Lancet 393.10172 (2019): 702-716.

70. $\mathrm{Xu} \mathrm{Y.,} \mathrm{et} \mathrm{al.} \mathrm{"Characterization} \mathrm{of} \mathrm{inflammatory} \mathrm{cytokine}$ profiles in cerebrospinal fluid of hand, foot, and mouth disease children with enterovirus 71-related encephalitis in Hangzhou, Zhejiang, China”. Medicine. 98.52 (2019): e18464.

71. Tansarli GS and Chapin KC. "Diagnostic test accuracy of the BioFire ${ }^{\circledR}$ FilmArray ${ }^{\circledR}$ meningitis/encephalitis panel: a systematic review and meta-analysis". Clinical Microbiology and Infection (2019).

72. Zhao WL., et al. Zhonghua Yi Xue Za Zhi. 98.15 (2018): 11521157.

73. $\mathrm{Hu} \mathrm{Z}$., et al. "Metagenomic next-generation sequencing as a diagnostic tool for toxoplasmic encephalitis". Annals of Clinical Microbiology and Antimicrobials 17.1 (2018): 45.

74. Liu LL., et al. "Next-generation sequencing technology as a powerful detection and semi-quantitative method for herpes simplex virus type 1 in pediatric encephalitis". Journal of NeuroVirology (2019).

75. Kennedy PGE., et al. "Viral Encephalitis of Unknown Cause: Current Perspective and Recent Advances". Viruses 9.6 (2017):138.

\section{Assets from publication with us}

- Prompt Acknowledgement after receiving the article

- Thorough Double blinded peer review

- Rapid Publication

- Issue of Publication Certificate

- High visibility of your Published work

Website: https://www.actascientific.com/

Submit Article: https://www.actascientific.com/submission.php

Email us: editor@actascientific.com

Contact us: +919182824667 